IRA International Journal of Management \& Social Sciences ISSN 2455-2267

Vol. 14, Issue 02 (Special Issue) pg. 145-156.

International Conference on Wellbeing: Lifespan Perspectives $\Xi^{\circ}$ Practices for Sustainable

Communities, 2019.

\title{
Is Resilience Affected By Parental Mental Illness? A Study of Tribal Young Adults
}

Gayathri Devadasan

Tata Institute of Social Sciences - Banyan Academy of Leadership in Mental Health, Chennai, India.

DOI: $10.21013 /$ jmss.v14.n2sp.p14

(c) Authors.

Type of Review: Peer Reviewed under the Responsibility of the conference's Scientific Committee.

Disclaimer: The copyright to this work is retained by the authorship. This work contains the opinions Ev views of the authorship solely and the same are not the views or opinions of the IRA. IRA disclaims of any harm or loss caused due to the published content to any party. 


\begin{abstract}
Resilience is a sign of positive psychological coping amidst adversity. Limited studies have investigated resilience in young adults who have a parent with mental illness, and fewer in tribal populations. This mixed methods study was based on a sequential explanatory model. A pilot field study was conducted with tribal young adults $(\mathrm{n}=10)$. The main study aimed to assess resilience in tribal young adults $(n=61)$ through the administration of the Tamil translated version of the Wagnild\& Young Resilience Scale (2009). Purposive sampling from a tribal hospital's records derived young adults from 4 vulnerable tribal communities residing in Nilgiri hills of South India. Target group comprised tribal young adults $(\mathrm{n}=31)$ who had a parent with depression or psychosis, and comparison group comprised tribal young adults $(n=30)$ with parents without mental illness. A subsample of participants from each group $(n=5+5)$ were selected for a semi structured interview. Quantitative and qualitative data were analysed using Independent Samples t Test and Thematic Analysis respectively. Results revealed that overall resilience was not significantly different between target and comparison groups, which accepted the null hypothesis. Resilience was not significantly different between females and males, which again accepted the null hypothesis. Despite living in severe adversity tribal young adults are resilient; parental mental illness is only one among many vulnerabilities faced by them. The prevailing extrinsic and intrinsic protective factors might explain their resilience.
\end{abstract}

Key Words: resilience, risk, young adults, youth,indigenous, culture,mentalhealth,coping

Adivasis or indigenous tribal people are characterised by their unique language, customs and cultural practices. Globally, theyhave a historical context of colonial invasion and rulingwhich brought dispossession and tremendous suffering (United Nations, 2009). The Indian government identifies scheduled tribes by theirprimitive traits, distinctive culture, shyness with the public at large, geographical isolation and social and economic backwardness(Indian Ministry of Tribal Affairs, 2004).Scheduled tribes fall low in education, health, social and economic indicators on the HDI (UN, 2009). Indian tribes are typically situated in poorer rural areas (Chattopadhyay, 2011).

Mental health is a state of well-being characterised by functionality and contribution to society (World Health Organization, 2003), while mental illness comprises behavioural or psychological syndromesreflecting dysfunctionand pathology (Butcher, 2014). Psychosis anddepressionfeature among the most prevalent mental disorders at the global level (Lehtinen\&Joukamaa, 1994).Mental illness affects people worldwide, particularlyvictims of abject poverty, abuse or violence, and tribal populations (WHO, 2003). It causes multiple dysfunctions in affected individuals, witheconomic loss and incalculable personal and familial suffering(APA, 2013).It has a direct impact on families, especially children. Stigma and discrimination often isolate such families from society, with caregiver burden and burnouttaking a toll. While families can help the treatment process and potentially promote recovery, they are often unable to.

Psychological factors play a vital role in precipitating and perpetuating mental illness. Theories suggest the impact of childhood experiences on later outcomes (Freud, 1905; Bowlby, 1982). Erik Erikson's theory(1950) proposed negative outcomes during any stage can be resolved in a future stage; a positive model accounting for resilience (Fleming, 2004).The Theory of Positive Emotions (Fredrickson, 1998;2001) proposed psychological coping is enhanced by positive emotions. Caregivers play a crucial role in promoting or neglecting a positive environment for children. When a parent has depression, the child is exposed to the parent's persistentnegative views about self, world and future (McGinn, 2000).Mak, Winnie \& S W Ng, Ivy \& Wong, Celia (2011) proposed a Positive Cognitive Triad; a positive view of self, world and future. Positive emotions have afleeting natureunlike negative emotions which persist; a negative bias. This had survival value in primitive hunting societies as it provided negative cuesfor threat in the environment (Fredrickson, 2004). However, this meant missing positive occurrences (Fredrickson, 2001). The capacity to retain positive emotions during stress may be indicative of higher resilience.

Resilienceisdefined asthe process of adapting well to adversity, trauma, tragedy, threats or significant sources of stress(APA, 2016). Most individuals experience and overcome adversity throughout their lifespan (WHO, 2013). The outlook that resilience is ordinary has positive implications unlike deficit-focused models suggesting adversity results in negative life outcomes or pathology (Hetherington and Blechman,1996). This offers the possibility of prevailing protective factors and resilience in children from disadvantaged backgrounds.

International Conference on Wellbeing: Lifespan Perspectives E Practices for Sustainable Communities, 2019. 
Figure 1 illustrates resilience and itsdynamic relationship with adversity, risk, vulnerability and protective factors. Adversity is exposure to harsh environmental influences (Rutter, 2012). Riskis a predisposition to a negative event arising from adversity, whilevulnerabilityincreases susceptibility to risk(Lacharité, 2005). Protective factorsreduce the impact of risk, thereby enabling individuals to overcome adversity. Steeling effectsare a decrease in vulnerability with exposure to adversity(Rutter, 2012). Adversity being a prerequisite for resilience implies that sheltering from adverse life events obstructsresilience.

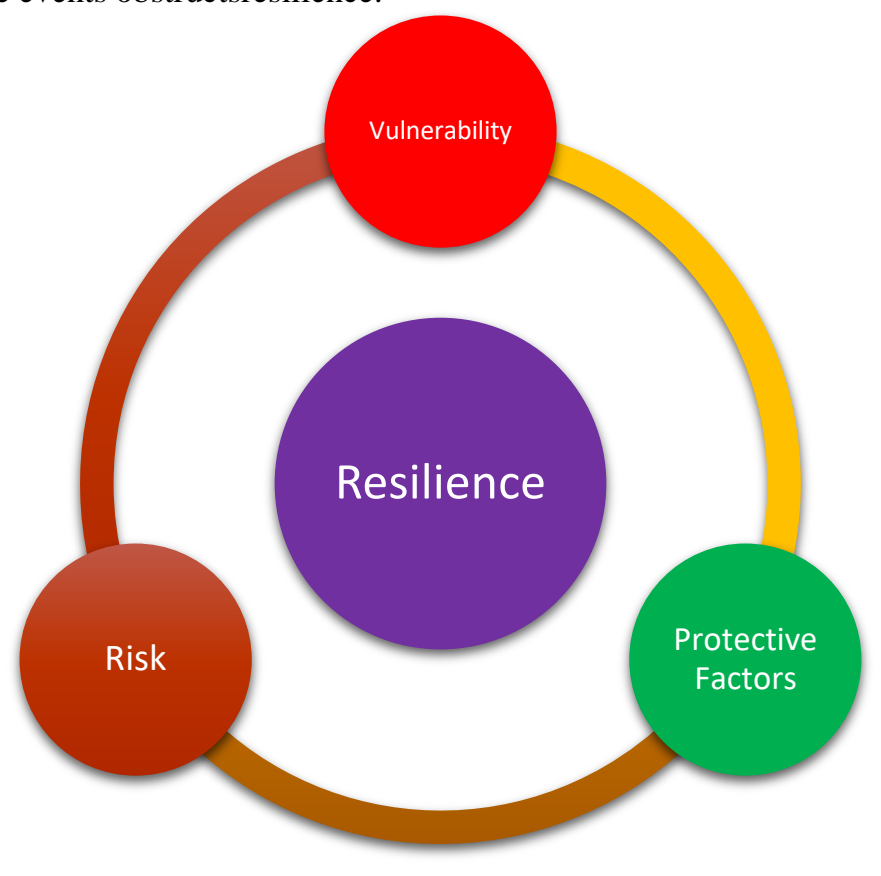

Figure 1 Resilience and interacting factors

Review of literature illustrates that mental health research is a neglected area (Mehta, Joseph \&Verghese, 1985). Parental mental illness is found to affect child mental health outcomes (Mustillo, Dorsey, Conover, \& Burns, 2011) with some individuals having the capacity to emotionally detach themselves from their affected parent (Anthony and Anthony (1987) while others are at greater risk for developing the same (Sullivan, Neale \& Kendler, 2000; Olfson, 2003). Resilience based research emerged in the 1970s, bringing a fundamental shift from deficitfocused to strength-based models (Masten, 2014). This broadeningscope of psychology threw light upon the positive potential in man. Resilience is found to haveuniquecultural aspects (Resilience Research Centre, 2005). Aboriginal research by Ledogar and Fleming (2008) revealed that perceived discrimination and historical trauma contribute to adversity in aboriginal communities through the world; while some aboriginal youth respond positively to adversity, others do not.

\section{Rationale}

Research on mental illness is largely neglected at national and international levels. There are few studies on mental health in indigenous communities, which primarily focus on physical health. Mental illness is associated with incalculable individual and family suffering and found to have a direct impact on intimate relationships, e.g. parentchild relationship.Children of parents with mental illness are at increased risk for mental health problems, which affects their psychological coping. The tribal population is vulnerable, hence tribal young adults with a parent with mental illness are possibly more vulnerable. Majority of studies focus on risk factors and vulnerability associated with psychopathology. Although the new trend is a shift from deficit-focused to strength-based models, there is still a dearth of the latter with few studies focusing on protective factors and resilience. Little is known about why some individuals at risk do better than others. Resilience based research mostly comprises studies on family resilience and theoretical papers with a conceptual orientation. There are limited studies addressing resilience in children, and fewer on the impact of parental mental illness on tribal children. The current study attempts to address these gaps and contribute to the existing body of knowledge. 


\section{Research Question}

Is there a difference in the resilience between young adults who have a parent with mental illness (depression or psychosis), and young adults who do not have a parent with mental illness?

Aims

- To examine the difference in resilience in tribal young adults who have a parent with mental illness and those who do not have a parent with mental illness

- To explore factors which promote and hinder the development of resilience

\section{Method}

\section{Research Objectives}

- To measure resilience in tribal young adults with one parent with Major Depressive Disorder or Psychosis

- To measure resilience in tribal young adults who do not have a parent with mental illness

- To explore socio demographic factors which influence resilience

- To explore psychosocial factors which influence the development and presence of resilience in young adults

- To identify significant life events which facilitate the development of resilience

- To identify vulnerabilities which influence the development of resilience

\section{Hypotheses}

Hypothesis-1 Resilience scores will not differ in young adults with a parent with mental illness and young adults without a parent with mental illness.

Hypothesis-2 Resilience scores will not differ in female young adults and male young adults.

Hypothesis-3 Resilience scores will not differ in married and unmarried young adults.

\section{Field of Study}

This study was conducted in Gudalur Valley, Nilgiris District, Tamil Nadu. It was carried out via the Mental Health Program of Gudalur Adivasi Hospital (GAH). GAH is run by a charitable trust, Association of Health Welfare in the Nilgiris (ASHWINI), which has provided comprehensive health care for the tribals of Gudalur Block for the past 27 years.

\section{Population}

The tribal population in the Nilgiris district consists of four tribes.Gudalur Block was selected due to its large tribal population, thereafter allowing more groups of tribes to be fairly represented. For this study 8 areas (21 villages) were visited.

\section{Sample}

For the main study, the target sample was obtained from the psychiatric in-patient and out-patient hospital records wherein screening for mental illness was conducted by a psychiatrist. The inclusion criteria constituted 'young adults' (age range operationally defined as 20-35 years) who 1) Had only one parent with depressive disorder or psychosis, 2) Belonged to a tribal community and did not have a parent with mental illness.Post pilot study the age range was expanded to include 17-35 year olds, upon discovery that there are many school dropouts who are a part of the working population. The comparison group was obtained from the community and matched on socio-demographics shared by the target group. Equal representation of all four tribes was attempted. Nonprobability sampling was used in the form of purposive sampling.

\section{Research Design}

The study used a sequential explanatory mixed methods design, which was conclusive and descriptive in nature, illustrated in Figure 2. The quantitative phase followed ex post facto design where young adults $(\mathrm{n}=61)$; both males $(n=30)$ and females $(n=31)$ were selected based on parental mental illness and the WYRS was administered on them. A subsample of participants from the quantitative phase $(n=10)$; both males $(n=4)$ and females $(n=6)$ were selected for the personal interview. Criteria for selection was based on extreme scores derived from the resilience scale which was administered on the target and comparison groups. The study duration was 3 months. 


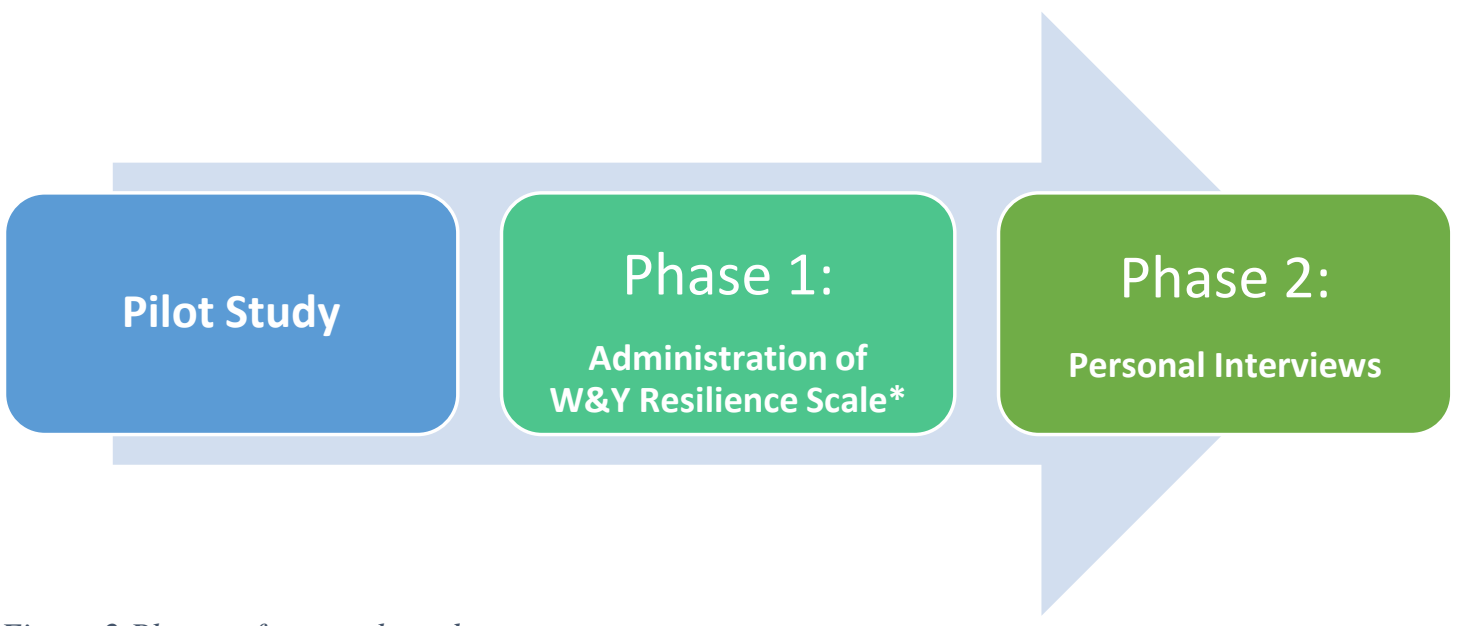

Figure 2 Phases of research study

A pilot study was conducted with a sample of 10 young adults. A significant finding from the pilot study was that administering the scale in written form took about an hour and required explanations which interfered with reliability; while an oral pre-recorded version took only about 10 minutes to administer with no interference. Hence the audio version of the scale was used for the main study.

\section{Tools}

To elicit info about participant's profile a Demographic Sheet was prepared, comprising factors including age, gender, siblings, marital status, children, education, occupation and tribe details.Resilience was measured using a standardized scale, namely Wagnild\& Young Resilience Scale, 2009*. The 7-point Likert scale consisted of 25 items. The Tamil adapted version of the scale, translated by Mr. Karthik Lakshmanan was used. The Cronbach's alpha coefficients ranged from 0.72 to 0.94 , supporting internal consistency reliability and construct validity (Wagnild, 2009). It has been used on a wide range of study populations, including individuals of different ages, socioeconomic and educational backgrounds (Wagnild, 2009). The semi structured interview obtained information regarding the psychosocial factors influencing the individual's coping and resilience, important psychological events through their lifespan, perceptions about their parent's mental illness. The Interview Guide was slightly modified post pilot study.

\section{Plan of Analysis}

Quantitative data was analysed using the Statistical Package for Social Sciences (SPSS 16.0). Independent Samples t Test was computed. Qualitative data was translated from Tamil and Malayalam into English, and simultaneously transcribed. Thematic Analysis was performed.

\section{Ethical considerations}

The hospital's ethics committee approved the study before data collection.Participants were briefed prior to the study and written informed consent (Tamil) was sought. They were sensitized and made aware of their rights. Participation was on a voluntary basis which could be withdrawn at any point during the study. There was a possibility of minimal psychological harm as sensitive personal information was derived from participants. When a participant experienced psychological distress, especially at times when s/he was unable to communicate it, the researcher addressed it by providing counselling or referring to a counsellor/psychiatrist in the hospital after providing psychoeducation for the same. All the data obtained from the resilience scale and interview process was coded and identifiers were removed. The data was kept strictly confidential and the only persons who had access to the data were the researcher and her guide. 


\begin{tabular}{|c|c|c|c|c|}
\hline $\begin{array}{l}\text { Results } \\
\text { Quantitative Resul } \\
\text { TABLE } 1 \\
\text { Sociodemographic }\end{array}$ & f sample & & & \\
\hline & Target $(\mathrm{N})$ & $\%$ & Comparison $(\mathrm{N})$ & $\%$ \\
\hline & 31 & $51 \%$ & 30 & $49 \%$ \\
\hline Females & 16 & $52 \%$ & $19 \%$ & $63 \%$ \\
\hline Males & 15 & $48 \%$ & $11 \%$ & $37 \%$ \\
\hline Age (Mean) & 24.16 & & 19.77 & \\
\hline Age (15-20 years) & 13 & $42 \%$ & 17 & $63 \%$ \\
\hline Age (21-35 years) & 18 & $58 \%$ & 13 & $37 \%$ \\
\hline Siblings (Mean) & 2 & & 3 & \\
\hline Unmarried & 17 & $55 \%$ & 17 & $57 \%$ \\
\hline Married & 14 & $45 \%$ & 13 & $43 \%$ \\
\hline Without children & 16 & $52 \%$ & 25 & $83 \%$ \\
\hline Have children & 15 & $48 \%$ & 5 & $17 \%$ \\
\hline EDUCATION & & & & \\
\hline NIL & 3 & $10 \%$ & 0 & $0 \%$ \\
\hline Secondary & 11 & $35 \%$ & 7 & $23 \%$ \\
\hline High School & 13 & $42 \%$ & 18 & $60 \%$ \\
\hline College & 4 & $13 \%$ & 5 & $17 \%$ \\
\hline OCCUPATION & & & & \\
\hline Doing nothing & 4 & $13 \%$ & 8 & $26 \%$ \\
\hline Homemaker & 9 & $29 \%$ & 11 & $37 \%$ \\
\hline Coolie & 11 & $36 \%$ & 4 & $13 \%$ \\
\hline Farmer & 1 & $3 \%$ & 2 & $7 \%$ \\
\hline Student/Skilled & 6 & $19 \%$ & 5 & $17 \%$ \\
\hline TRIBE & & & & \\
\hline Paniya & 23 & $74 \%$ & 11 & $37 \%$ \\
\hline Bettakurumba & 1 & $3 \%$ & 11 & $37 \%$ \\
\hline Kattunayakka & 5 & $16 \%$ & 4 & $13 \%$ \\
\hline Moolakurumba & 2 & $7 \%$ & 4 & $13 \%$ \\
\hline
\end{tabular}

Table 1 illustrates the socio-demographic composition of the sample. The sample comprised the target group and comparison group; target group $(\mathrm{n}=31)$ constituted tribal young adults with a parent with mental illness, and the comparison group $(n=30)$ comprised tribal young adults with parents without mental illness. There were roughly equal numbers of men and women within an age range of 20-25 years. While both groups have about same number of married individuals the target group is of a higher age group with more children. Most individuals attended secondary or high school with few unschooled and college going individuals. Majority were kooli workers or homemakers with some students and skilled professionals. Composition of different tribes was skewed with Paniyas predominating.

TABLE 2

Resilience in tribal young adults with and without a parent with MI

\begin{tabular}{cccccc}
\hline Group & $\mathrm{N}$ & Mean & SD & t value & $p$ value \\
\hline Target & 31 & 113.61 & 26.16 & -1.21 & 0.23 \\
Comparison & 30 & 121.52 & 24.41 & & \\
\hline
\end{tabular}

Table 2 illustrates the difference in resilience between Target Group; young adults with a parent with mental illness, and Comparison Group; young adults with parents without mental illness. From the above table it is inferred that there is no difference in resilience between the target group and the comparison group, $\mathrm{t}=-1.21, p$ $>0.05$. 
TABLE 3

Resilience in young adults with a parent with depression and young adults with a parent with psychosis

\begin{tabular}{|c|c|c|c|c|c|}
\hline Target Group & $\mathrm{N}$ & Mean & $\mathrm{SD}$ & $t$ value & p value \\
\hline $\begin{array}{l}\text { Parent with } \\
\text { depression }\end{array}$ & 14 & 114.86 & 28.21 & \multirow{2}{*}{0.23} & \multirow{2}{*}{0.82} \\
\hline $\begin{array}{l}\text { Parent with } \\
\text { psychosis }\end{array}$ & 17 & 112.59 & 25.18 & & \\
\hline
\end{tabular}

Table 3 illustrates the difference in resilience between young adults who have a parent with depression and young adults who have a parent with psychosis. From the above table it is inferred that there is no difference in resilience between young adults who have a parent with depression and young adults who have a parent with psychosis, $\mathrm{t}=0.23, p>0.05$.

TABLE 4

$t$ Test results for differences in resilience across sociodemographic factors

\begin{tabular}{ccccccc}
\hline Groups & Groups & $\mathrm{N}$ & Mean & SD & t value & $p$ value \\
\hline \multirow{3}{*}{ Gender } & Females & 35 & 119.46 & 23.12 & & \\
& Males & 26 & 115.42 & 28.33 & 0.59 & 0.56 \\
\multirow{5}{*}{ Age } & $15-20$ years & 30 & 116.37 & 26.13 & & \\
& 21-35 years & 31 & 119.06 & 24.88 & & \\
\multirow{3}{*}{ Marital Status } & Unmarried & 34 & 119.50 & 25.58 & & 0.68 \\
& Married & 27 & 115.52 & 25.32 & & \\
& & & & & & \\
\hline
\end{tabular}

Table 4 illustrates the resilience in 1) Female and male young adults, 2) Young adults between 15-20 years \& 21-35 years, and 3) Unmarried and married young adults. It is inferred from the above table that there was no significant difference in resilience scores between female and male young adults; young adults between 15-20 years $\&$ 21-35 years; and unmarried and married young adults: $\mathrm{t}=0.59, p>0.05 ; \mathrm{t}=-0.41, p>0.05 ; \mathrm{t}=0.55, p>0.05$.

\section{Qualitative Results}

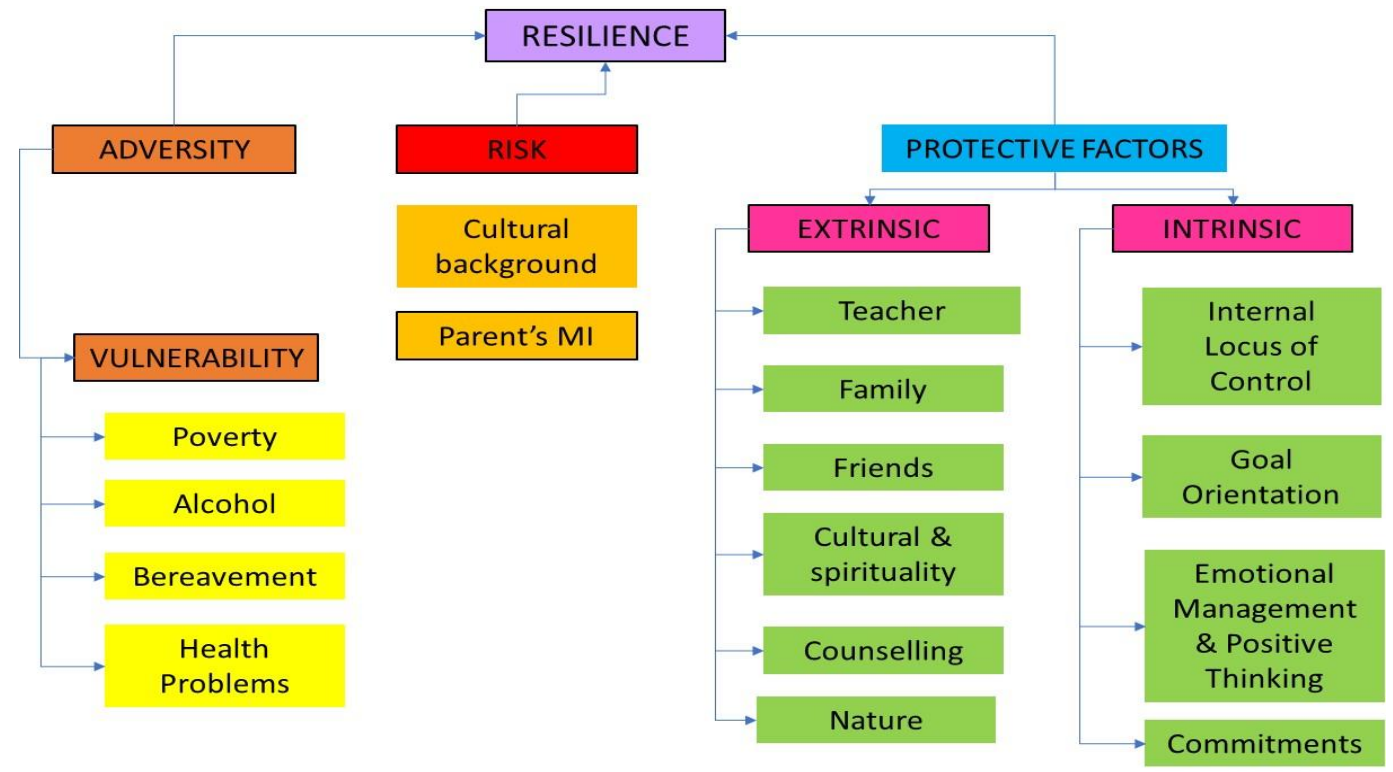

Figure 3 Factors influencing resilience 
Figure 3 lists factors found to influence resilience. Participants' narratives described common vulnerabilities exposed to since birth, with poverty and alcohol abuse by family member/s being major sources of distress at individual, familial and societal levels. The interacting effects of vulnerabilities emerged with description of daily struggles and changing life when a parent/s left them with another relative, fell ill or died. Being abandoned by parents without a replacement caregiver was often followed by pain of loss and unmet physical, psychological, financial and educational needs which furthered poverty. Some cultural aspects impeded availing equal opportunities in higher education, command of English and employment. Some felt inferior. Limitations were experienced despite hard work and constant efforts, which was demotivating.

Regarding parental mental illness, the parent's domestic unproductivity, impaired occupational functioning, lack of parenting and emotional unavailability had a direct impact on the individual's life. They often took on additional work and became caregiver to their mentally ill parent. Financial issues due to parental unemployment had detrimental effects on the individual's health, quality of life, and avenue for opportunities. However, there was no mention of stigma, shame, social exclusion or homelessness (generally associated with mental illness). The parent with mental illness was not neglected or rejected by family. Individuals did not blame the parent but attributed their behaviour to the illness.

Internal and external protective factors helped individuals enjoy life and promoted resilience in difficult circumstances. Even one significant relationship aided coping during adversity. Positive teacher experiences (school/college) were a recurrent theme in all interviews. Teachers provided academic, emotional, and financial support for which gratitude was expressed. They helped them by using non-punitive teaching methods which eliminated their fear of learning; repeating concepts till they understood; instilling interest in subjects; and providing verbal positive reinforcement which boosted self-esteem. Teachers became a positive role model who they aspired to be to the extent that some have taken up teaching as a profession. Those who sought counselling at the hospital received emotional catharsis and clarity.

Family constituted members blood related/ informally adopted/related by marriage, while community contained nuclear and extended families. The tribal population lives in clusters on hills with some small villages comprising an entire extended family. This geographical proximity often translated into emotional closeness. Family was a source of support and enjoyment, especially when parents were unavailable during childhood. Meeting cousins and friends were joyful times and these connections remain important. Relatives coming home and staying during festivals was associated with happiness while sadness was associated with time for their departure.

Tribal culture is deeply embedded in nature. Customs practiced till today such as joyful singing and dancing in circles during festivals fostered a deep sense of belonging. This faith in belonging to a larger 'world' encompassing nature helped provide meaning in times of adversity. Time in nature also alleviated stress, inducing relaxation. Their understanding of nature also put some at an advantage for certain subjects in school/college.

In terms of internal protective factors, academic and personal struggles were overcome by consistent efforts, internal motivation and goal setting; following which, improvement heightened self-esteem and fuelled greater efforts. For those funded by the organisation for further studies monetary support was instrumental in helping them persevere and achieve goals. Commitments were made to self, family, or community, and these operated as motivating factors to success.

Emotional management among males and females constituted expression of emotion (sharing with someone close, crying), logical reasoning/problem solving, and distraction techniques. Resilience was evident in an acceptance of their life circumstances, moments of bravery and victory, and concrete personal changes leading up to positive personal growth. All of these had a beginning in a significant adverse event in the individual's life.

\section{Discussion}

The consequences of historical displacement and marginalisation of tribal societies was evident in this study, as is globally (UN, 2009; Ledogar and Fleming, 2008). Adversity presented itself as alcohol related issues, health problems, incomplete education andunemployment, in consensus with existing literature (UN, 2009). Despite this host of vulnerabilities resilience has the potential to emerge amidst adversity (WHO, 2013). Adversitywas perceived to bea part of life's journey in this study, with struggle being seemingly choiceless and resilience 
providing the bandwidth to engage with it.Hence, as a psychosocial risk experience adversity is a prerequisite for the manifestation of resilience (Ong, Bergeman\& Chow, 2010). This perspective, from strength-based models offers prevailing protective factors and resilience in children from disadvantaged backgrounds(Hetherington,1996).

The primary finding from this study is that resilience is average in this vulnerable population; it is neither low nor high. There were no statistically significant interactive effects when testing resilience with sociodemographic variables, which goes against a previous study (Rutter and Quinton, 1984). There was no difference in resilience between tribal young adults with and without a parent with mental illness, which has important implications. It questions deficit-focused models which emphasise an optimal environment during childhood as a necessity for the development of future positive outcomes. Since resilience scores are indicative of an individual's ability to cope with life stressors, they also determine their risk for mental illness; an average resilience score implies these individuals are at no more risk than the average population for the development of mental health issues, contrary to previous findings (NCH, 2007). It also refutes the claim that vulnerable groups are at increased risk for the development of mental disorders (WHO, 2003).

Mental illness is debilitating, causing multiple dysfunctions in affected individuals (APA, 2013). Occupational and economic effects plus caregiver burden were highlighted in this study. Parental mental illness was not viewed as an issue in isolation; it was the consequences of the absence of that parent's functioning which affected the young adult at a survival level. Stigma and social exclusiongenerally associated with mental illness did not feature.Does this imply that there is less stigma and discrimination towards mental illness in this community? This may provide further insights into the relationship between shame and resilience, wherein shame is found to be an impediment to the presence of resilience (Brown, 2004), suggesting differences in the experiencing of mental illness. Individuals did not blame the parent but attributed their behaviour to their illness. This was empowering as it allowed them to accept the situation and cope but disempowering as they felt unable to improve things by seeking mental health services, which may be due to low awareness.

So, what is it about this vulnerable population which makes them resilient? Extrinsic and intrinsic protective factors cultivated resilience, as supported by literature (Daniel \&Wassell, 2002). Resilience is fostered by the presence of supportive relationships (Kring et al, 2007). Even one significant close relationship made for positive experiences and coping during difficult times. Positive teacher experiences nurture resilience (Werner, 1995). Teachers provided emotional support and believed in the young adult's potential. They filled the loss of a functioning parent by being a 'positive parent' or role model.Family, marital and community support also served as a secure base and anchor for resilience, not in-keeping with other findings (Somers, 2007). A slower paced, traditional lifestyle with cultural practices involving music and dance encompassed the spirit of community support. Traditional activities and spirituality were protective as they established a degree of predictability in life. Counselling services were availed and beneficial during distressing times. An ever-present and almost missed protective factor in the lives of this population was nature itself. Time in nature was found to have therapeutic effects which stimulated resilience, contrary to previous findings where parental mental illness kept children indoors (Somers, 2007).

Various internal psychological mechanisms contribute to the promotion of resilience and not a specific trait (Luthar et al, 2003), as apparent in this study. Emotional management is the ability to express emotion in a genuine and healthy manner. The experiencing of different intense emotional states may or may not be an indicator of resilience; with the presence of negative emotions not always implying inability to cope. In the current study participant's narratives, laughter and playfulness reflected positive traits and thoughts (Fredrickson, 1998;2001; Lee et al, 2013; Olsson et al, 2003).Individuals had a "Positive Cognitive Dyad"; a positive view of themselves and sometimes of the future, however they did not have a positive view about the world, especially regarding alcohol problems in their family and community and hence did not possess a Positive Cognitive Triad (Mak et al, 2011). Positive experiences which instilled optimism in childhood had enduring effects lasting into adulthood wherein these positive memories helped individuals cope during difficult times, as supported by literature (Leela et al, 2013; Seligman, 1990).

Resilient individuals possessed a basket of protective factors; 1) An adult who believed in their potential; a teacher or someone in the organisation who offered emotional/academic/financial support, 2) An internal locus of 
control; a belief that they can achieve goals with their own efforts 3) Goal orientation; an individual disposition towards goal setting and achievement, and 4) Commitments; personal, familial, or organisational.

Perceived choicelessness and survival instinct protected young adults by motivating them during adversity. Hope remained. An acceptance of life circumstances appeared to be a precondition for resilience, following which authentic engagement with adversity was made possible. Internal motivation aided their efforts and brought success, with increased perceived control for achievement. Thus, adversity did not merely set stage for resilience, it catalysed psychological transformation alongside protective factors. All these facets together hold evidence for resilience in this vulnerable community.

\section{Limitations}

The sample was derived from tribal hospital records which may not be representative of the entire tribal population, although this is the only tribal hospital in the region. All four tribes were not equally represented. Matching between groups for socio-demographics was not performedfor all categories.Travel time to remote villages was long and time at site limited. Completion of scale administration was given more priority to meet basic statistical requirements of sample size. Resilience scale (Wagnild, 2009) results may not accurately reflect resilience in sample due to low educational level, difficulty in abstraction and rating the 7-point Likert scale, unfamiliarity with psychological testing process, discomfort interacting with unfamiliar researcher. Regarding the interview process, rapport building was difficult and time consuming in this culturally shy community as the researcher was an 'outsider'. Limited schooling and a low development of metacognition may account for low emotional literacy which made it difficult for participants to elaborate their emotional experiences. The researcher's interviewing skills evolved through the research process. The researcher was initially afraid of participants having an emotional breakdown due of 'ethical baggage', however post research supervision the researcher became aware that she is trained to provide psychological first aid if required.

\section{Implications and Future Prospects}

This study addresses the need to strengthen existing extrinsic and intrinsic protective factors prevailing in individuals, thereby making for better psychological coping. It highlights the critical role, potential andlasting impact of teachers and the education system in fostering resilience in the lives of young people. The finding that an audio version of the resilience scale can be comprehended significantly faster by an illiterate population in comparison to a paper version has important implications inresearch, and the fields of education and psychology. Findings of this study cannot be generalised to other tribal populations due to this community's unique cultural aspects, or to the rest of the Indian population because of the country's geographical, linguistic and ethnic diversity (Math, Chandrashekar \&Bhugra, 2007).

This study can be replicated as a comparison between tribal and non-tribal young adults, or with qualitative methods using an immersive approach. Emotional intelligence and gender differences can be explored.Absence of stigma, shame, discrimination and social exclusion towards mental illness can be investigated, focusing on prevailing culture-specific protective factors. The relationship between poverty, locus of control and goal orientation in underprivileged populations can be studied.Action research with community awareness and family therapy for alcohol abusers can be conducted in a community reporting high alcohol abuse.

In conclusion, it can be said that having a parent with mental illness is only one among the multiple risks and vulnerabilities faced by tribal young adults. Resilience is born in adversity. Tribal young adults used the minimal materialistic resources they had, took the accessible opportunities, and worked hard to do well in school and college. They evolved into competent, independent young adults. The Gudalur Adivasi Hospital's contributions in terms of provision of 1) Health and mental health services to the community, 2) Opportunities and funding for further studies, and 3) Guidance and mentoring by staff members cannot be overlooked as factors which promoted resilience in these young adults. Finally, it is important to note that the tribal community is not only filled with adversity, there are intrinsic and extrinsic protective factors which make for resilience. The tribal people have 'a symbiotic relationship with nature, minimalistic living and an egalitarian culture' (Subramanian, 2006). This way of life is often therapeutic and healing. 


\section{Acknowledgements}

The researcher feels deeply grateful to all the staff at Gudalur Adivasi Hospital for permitting and helping her conduct this study. It is important to note that the study built on GAH's pre-existing rapport with the tribal community, without which accessibility to this population would be but a dream.

\section{References}

[1]. American Psychiatric Association. (2013). Diagnostic and statistical manual of mental disorders (5th ed.). Arlington, VA: American Psychiatric Publishing.

[2]. American Psychological Association (APA). (2016). Apaorg. Retrieved 27 July, 2016, from http://www.apa.org/helpcenter/road-resilience.aspx

[3]. Butcher, J.M. (2014). Abnormal Psychology: An overview. (Ed), Abnormal Psychology (pp. 5). New Delhi: Pearson Education.

[4]. Chattopadhyay, Aparajita. (2011). Urban versus Rural Poverty in India. Economic Affairs. 56. 225-232.

[5]. Fleming, J. (2004). Erikson's Psychosocial Developmental Stages. Retrieved 26 February, 2017, from http://swppr.org/textbook/ch 9 erikson.pdf

[6]. Fleming, J., \& Ledogar, R. J. (2008). Resilience, an evolving Concept: A review of literature relevant to aboriginal research. Pimatisiwin, 6(2), 7-23.

[7]. Fraser, E., \& Pakenham, K. I. (2009). Resilience in children of parents with mental illness: Relations between mental health literacy, social connectedness and coping, and both adjustment and caregiving. Psychology, Health \& Medicine, 14(5). Retrieved from https://doi.org/10.1080/13548500903193820

[8]. Fredrickson, B. (2004). The Broaden and Build Theory of Positive Emotions. University of Michigan. The Royal Society: 359: 1367-1377.

[9]. Fredrickson, B. (2011). The role of positive emotions in positive psychology. The broaden-and-build theory of positive emotions. The American psychologist, 56(3), 218-26.

[10]. Hetherington \&Blechman. (1996). Stress, Coping, and Resiliency in Children and Families. New York: Psychology Press.

[11]. India Ministry of Tribal Affairs (2004). The national tribal policy (draft). New Delhi: India Ministry of Tribal Affairs. India Ministry of Tribal Affairs. The national tribal policy (draft) New Delhi India Ministry of Tribal.

[12]. Kring et al. (2007). Abnormal Psychology. (10 ed.). United States: John Wiley \& Sons, Inc.

[13]. Lacharité, C. (2005). From risk to psychosocial resilience: conceptual models and avenues for family intervention (Unpublished master's thesis). Université du Quebéc, Quebéc, Canada.

[14]. Lehtinen\&Joukamaa. (1994). Epidemiology of depression: Prevalence, risk factors and treatment situation. Acta PsychiatricaScandinavica, 89(377), 7-10.

[15]. Luthar, S. S., Sawyer, J. A., \& Brown, P. J. (2006). Conceptual Issues in Studies of Resilience: Past, Present, and Future Research. Annals of the New York Academy of Sciences, 1094, 105-115. http://doi.org/10.1196/annals.1376.009

[16]. Mak, Winnie \& S W Ng, Ivy \& Wong, Celia. (2011). Resilience: Enhancing Well-Being Through the Positive Cognitive Triad. Journal of counseling psychology. 58. 610-7. 10.1037/a0025195.

[17]. Masten, A. S. (2014). Global Perspectives on Resilience in Children and Youth. Journal of Child Development, 84(1), 6-20. doi:10.1111/cdev.12205

[18]. Masten, Ann S. (2001). Ordinary magic: Resilience processes in development. American Psychologist, Vol 56(3), Mar 2001, 227-238. Abstract retrieved from PsycARTICLES database (PsycINFO Database Record (c) 2016 APA.

[19]. Math S., Chandrashekar C., \&Bhugra D. (2007). Psychiatric epidemiology in India. Indian Journal of Medical Research, 126(2), 183-192.

[20]. Mcginn. (2000). Cognitive behavioural therapy of depression: Theory, treatment, and empirical status. American Journal of Psychotherapy, 54(2), 0002-9564. 15

[21]. Mustillo, S. A., Dorsey, S., Conover, K., \& Burns, B. J. (n.d.). Parental depression and child outcomes: the mediating effects of abuse and neglect. Journal of Marriage and Family, 71(1), 164-180.

[22]. Resilience Research Center. (2005) International Resilience Project. Retrieved from http://www.resilienceresearch.org/research/projects/international-resilience

[23]. Rutter, M. (2012). Resilience as a dynamic concept. 24(2):335-44.

[24]. Rutter, M., \& Quinton, D. (1984). Parental psychiatric disorder: effects on children. Psychological medicine, 14(4), 853-80.

[25]. Somers, V. (2007). The impact of parental illness on children. The British Journal of Social Work, 37(8), 13191334.

[26]. Subramanian, S., Smith, G., Subramanyam, M. (2006) Indigenous health and socioeconomic status in India. PLoS Medicine: 3 (10), 1794-1804. 
[27]. United Nations. (2009). The concept of indigenous peoples. In DESA(Ed), State of the World's Indigenous Peoples (pp. 4-6). New York: United Nations Publication.

[28]. Werner, E. E. (1995). Resilience in development. Current Directions in Psychological Science, 4(2), 81-85. Retrieved from https://doi.org/10.1111/1467-8721.ep10772327

[29]. WHO. (2003). Investing in mental health (ISBN 924156257 9). Retrieved from Nove Impression website: http://www.who.int/mental_health/media/investing_mnh.pdf

\section{ABOUT THE AUTHOR}

Gayathri Devadasan is working as a counselling psychologist in private practice. She is also a consultant at Couch, Augmenta Health, in Bangalore. This research was conducted in collaboration with the Department of Applied Psychology in TISS-BALM, Chennai, as part of her Master of Arts degree in Applied Psychology with a specialisation in Clinical Psychology. 